\title{
UAS FOR GEO-INFORMATION: CURRENT STATUS AND PERSPECTIVES
}

\author{
R.B. Haarbrink \\ Miramap BV, Houten, the Netherlands - rhaarbrink@miramap.com
}

Commission I, WG I/V

KEY WORDS: UAV, UAS, Sensors, Photogrammetry, Lidar, Geo-Information, Regulation, Certification

\begin{abstract}
:
Recent and ongoing developments of state-of-the-art sensor technologies have resulted in smaller and lighter photogrammetric cameras with IMU, lidar scanners and other sensors that can now be integrated with and mounted on the larger Light UAS. This paper describes as an example the successful automatic flight of the 50-megapixel DigiCAM with AEROcontrol IMU developed by IGI flown on Geocopter's GC-201 unmanned helicopter system. The operational and technical requirements of UAS defined in new legislation remain however the safeguard to protect people and costly sensor payload assets. The current prospects of UAS-g face additional challenges related to end-user awareness and the return on investment. The current status of UAS legislation is given in this paper. This legislation justifies UAS-g operations of mapping coastal zones, forests, agricultural fields, and open mines. Sooner return on investment happens when the UAS legislation will be opening up airspace over urban areas (Class 2 approved UAS-g), to longer distances (BLOS operations), and to higher altitudes. UAS-g flights then become feasible to the maximum extent for cadastral mapping of larger areas, oil and gas pipeline monitoring, power line surveys, dike inspection, and highway and railway mapping.
\end{abstract}

\section{INTRODUCTION}

\subsection{Definition of UAS-g}

When a group of ten-year old kids was asked: "What can be done with a UAV?" the children responded:

$\checkmark$ Grocery shopping;

$\checkmark \quad$ Look in the neighbourhood who has a swimming pool;

$\checkmark$ Order a Happy Meal and deliver it warm;

$\checkmark \quad$ Walk your dog.

Genius applications of course, but there were also more serious answers. One of them was to perform measurements from the air. That is, Unmanned Aircraft Vehicle for geodetic and geotechnical survey flights (UAV-g). Or more correctly Unmanned Aircraft System for geodetic and geotechnical survey flights (UAS-g), since the Vehicle is always controlled and monitored by a Pilot Station on the ground and together they form the System.

\subsection{Military and Civil Market}

Many studies have proven that the UAS market is the fastest growing segment in the aerospace industry. UAS have initially been developed for military applications. When President Obama directed Predators UAS to Libya on 21 april 2011, General Cartwright explained the benefits of unmanned, armed UAS: “...their ability to get down lower, therefore to able to get better visibility on particularly targets now that have started to dig themselves in to defensive positions." General Cartwright said: "They're uniquely suited for urban areas where you can get low collateral damage, and so we're trying to manage that collateral damage, obviously, but that's the best platform to do that with."
Military UAS can be sold for a higher price and much of the defence industry is accustomed to a relationship with its governmental customers that are very different from that which is common in the non-military commercial sphere. Small- and medium-sized enterprises (SME) are best placed to exploit new markets outside the military UAS market, but large amounts of capital is required to develop UAS specific to the civil and commercial market. There is a trend that military and civil aviation authorities are nowadays working more closely together to harmonize the technical and operational standards of both worlds.

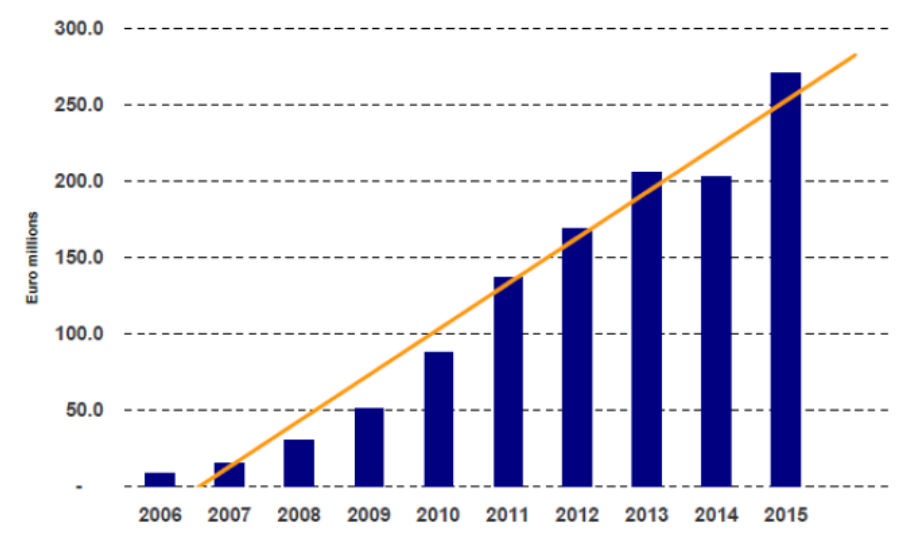

Figure 1. Total market for civil and commercial UAS markets in Europe (Frost \& Sullivan)

According to studies about the development of the Small and Light UAS market - that is UAS with maximum take-off mass (MTOM) up to $150 \mathrm{~kg}$ - this market will grow at rates of $30 \%$ per year and reach a value of about 250 million EUR in 2015 
(Figure 1). In October 2009 the European Commission (EC) organized a hearing on Small and Light UAS with the participation of SMEs from the European Community. This demonstrates the EC's high interest in the development of SMEs and their entry into this market (EC, 2009).

\section{CURRENT STATUS OF UAS-G}

\subsection{Benefits of UAS-g}

For mapping updates of smaller areas of 10 up to 100 hectares, there are various benefits of an autonomous UAS-g over traditional airborne survey flights. UAS-g also has great potential to become a good alternative to terrestrial geodetic surveys, especially to risky survey work along highways and train tracks, and other dangerous or inaccessible terrain (Haarbrink and Eisenbeiss, 2008).

$\checkmark \quad$ UAS-g operations reduce human life exposure in long air missions;

$\checkmark$ They provide economic savings and environmental benefits with less fuel consumption, less $\mathrm{CO} 2$ emission, and less noise compared to manned aircraft;

$\checkmark \quad$ The mobilization costs to the project area are lower and the UAS-g can be quicker deployed;

$\checkmark \quad$ The UAS-g acquires good quality aerial data under thin clouds, as the flying speed is slower and the average flying height above ground level (AGL) is lower. This also increases the average "clear sky" days per year to an estimated five times more operational days per year for UAS-g data collections;

$\checkmark \quad$ The digital imagery from 100 meters AGL result in very high resolution data, for example 1-3 cm pixel size using calibrated small or medium format digital cameras. From the imagery a Digital Surface Model (DSM) is extracted of approximately 50 points per $\mathrm{m} 2$;

$\checkmark \quad$ The operational costs of a UAS-g are low, depending on the system up to 550 euros per flight hour of the heavier vehicles all inclusive; and

$\checkmark \quad$ the UAS pilot training is relatively easy and accessible to any talented engineer compared to getting and maintaining a costly Commercial Pilot License that is required for manned airborne surveys.

\subsection{UAS-g Market Trends}

On the UAS-g market one can at the moment distinguish two separate groups.

The first group is covered by Small UAS with a Maximum Take-Off Mass (MTOM) of up to 25 kilograms. This class undergoes a successful market entry - especially the vehicles up to a few kilograms - because many users including universities, institutes, and engineering companies have decided to getting used in their organization with UAS-g operations. The price range of these small systems varies up to approximately 100,000 euros per system. The main drawback of these smaller systems is the limited flight time, limited useful payload, and thus the limited sensor quality. The question is whether these systems can produce the same quality geodetic products with the same accuracy levels as acquired by manned airborne system and terrestrial surveys as required by mapping agencies and mapping standards.
The second group is covered by larger vehicles with a maximum take-off mass up to $150 \mathrm{~kg}$, also called Light UAS. These systems are able to carry state-of-the-art sensors similar to the ones used in manned aerial survey missions, like for example medium-format digital cameras, and small lidar scanners. Most users including universities, institutes, and engineering companies are now moving towards UAS-g operations flying these heavier vehicles. The price range of the larger systems varies up to approximately 500,000 euros per system.

\subsection{UAS-g / Sensor Combinations}

Recent and ongoing developments of state-of-the-art sensor technologies have resulted in smaller and lighter photogrammetric cameras with IMU, lidar scanners (Eisenbeiss, 2009), hyperspectral sensors, passive microwave radiometers, thermal infrared cameras, video gimbal systems with real-time geo-location and even mini-SAR systems that can now be integrated with and mounted on the larger Light UAS.

\subsection{Medium-Format Camera with IMU on Light UAS}

An example of a new UAS-g development is the successful automatic flight of the 50-megapixel DigiCAM with AEROcontrol IMU developed by IGI flown on Geocopter's GC-201 unmanned helicopter system (Figures 2 and 3). This fully automatic photo flight was performed on 9 June 2011 in the Netherlands.

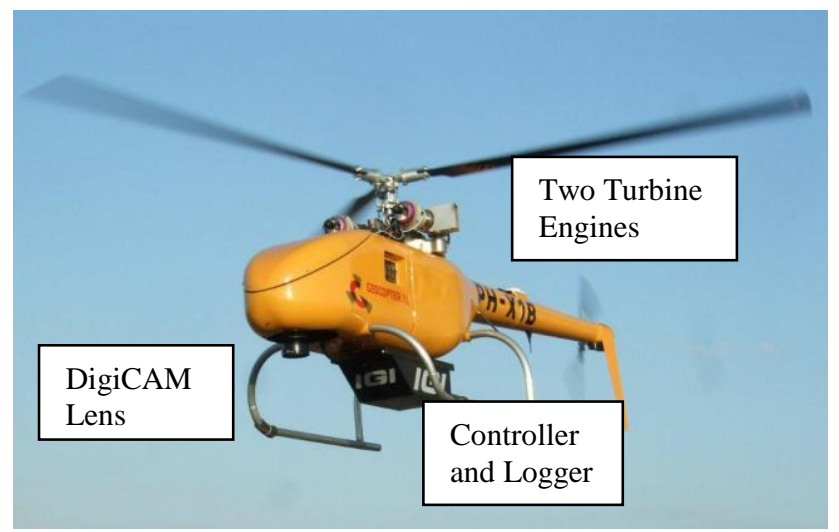

Figure 2. Geocopter GC-201 UAS-g with IGI DigiCAM and AEROcontrol

The total payload weight of the IGI components is approximately 10 kilograms. The complete system can be airborne for over one hour of high resolution data acquisition.

The DigiCAM is a medium-format airborne digital camera system for professional digital aerial photography. This digital camera is built for airborne conditions and aerial survey applications. Combined with IGI's AEROcontrol GPS/IMU system, DigiCAM forms a complete solution for an extremely rapid and automated workflow for the generation of directly georeferenced images.

AEROcontrol is the GPS/IMU system for precise determination of position and attitude of the projection center and the angles omega, phi, kappa of the DigiCAM. The AEROcontrol system consists of an Inertial Measurement Unit (IMU-IIe) based on fiber-optic gyros and a Sensor Management Unit with integrated high end GPS receiver. 


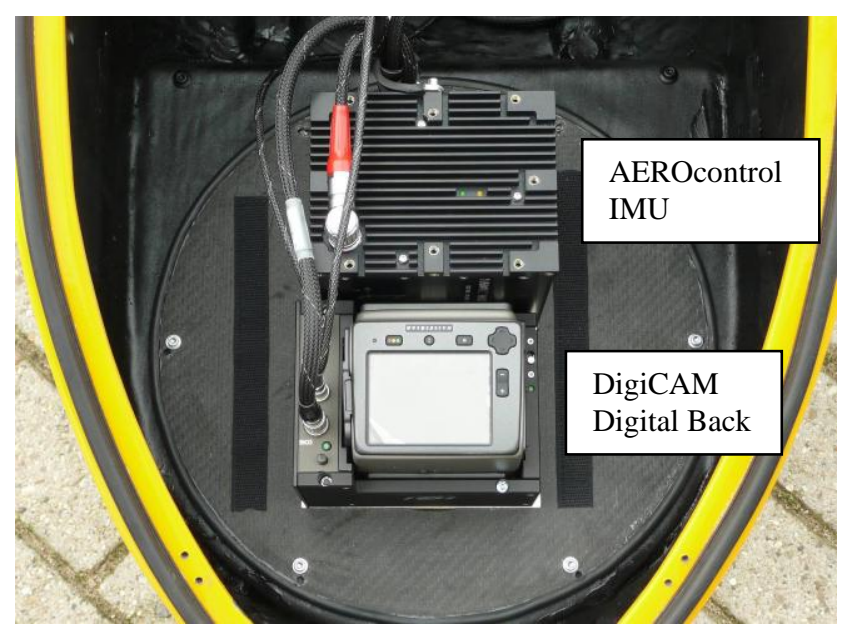

Figure 3. Sensor Installation in Geocopter GC-201 Payload Bay

The Geocopter GC-201 is an operational unmanned helicopter system with $90 \mathrm{~kg}$ MTOM and $3.3 \mathrm{~m}$ rotor span. The autopilot supports mission mode for pre-programmed survey flights including automatic triggering while recording the event file of the photo positions. Survey flights can be aborted and reprogrammed at any time during flight with simple keyboard control from the pilot station. The system features:

$\checkmark \quad$ Automatic take-off, mission and landing

$\checkmark \quad$ Two turbine engines running on Jet-A1 with One Engine Inoperative (OEI) capability

$\checkmark$ Ability to lift $30 \mathrm{~kg}$ state-of-the-art sensors and high definition camera systems

$\checkmark \quad$ Flight up to 1.5 hours

$\checkmark$ Flight approval Class 1 from the Dutch Civil Aviation Authorities (CAA)

The specifications of the integrated GC-201 with DigiCAM and AEROcontrol solution are given below in Table 1.

\begin{tabular}{|l|l|}
\hline System Parameter & Value \\
\hline UAS-g & Geocopter GC-201 \\
\hline Number of Engines & Two Turbine Engines \\
\hline Maximum Take-Off Mass & $90 \mathrm{~kg}$ \\
\hline Camera Triggering & Automatic by Autopilot \\
\hline Camera & IGI DigiCAM 50/60 Megapixel \\
\hline IMU & IGI AEROcontrol \\
\hline Project Parameter & Value \\
\hline Focal Length & 35 Millimeter Lens \\
\hline Flying Height & 50 Meters AGL \\
\hline Forward Overlap & $80 \%$ \\
\hline Sidelap & $60 \%$ \\
\hline Flight Lines & 4 Parallel and 4 Perpendicular \\
\hline Ground Sample Distance & 8 Millimeters (!) \\
\hline
\end{tabular}

Table 1. System and Project Specs

Four flight lines were flown parallel to the runway and four lines were flown perpendicularly (Figure 4 ). The photo mission was flown at 50 meters AGL which resulted in a stunning 8-mm ground sample distance. As one can observe the image has excellent radiometry - although it was a late evening flight with large shadows - and negligible image motion (Figure 5).

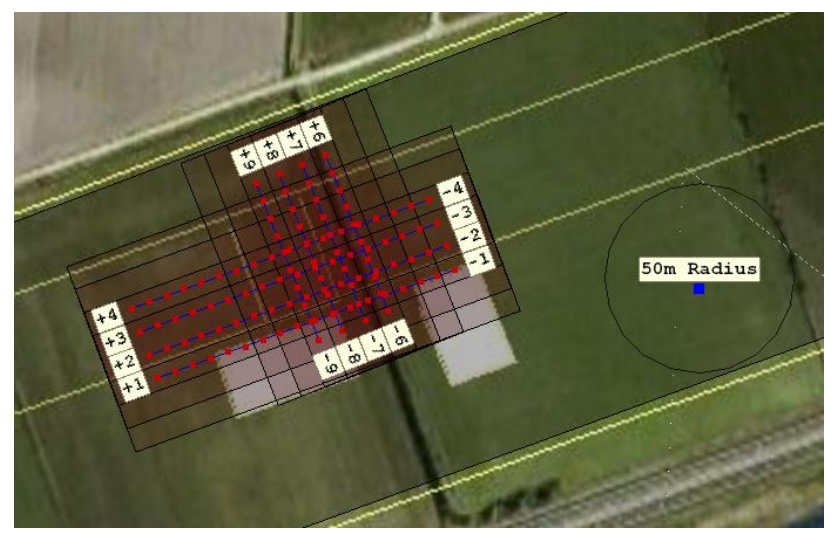

Figure 4. Flight Plan

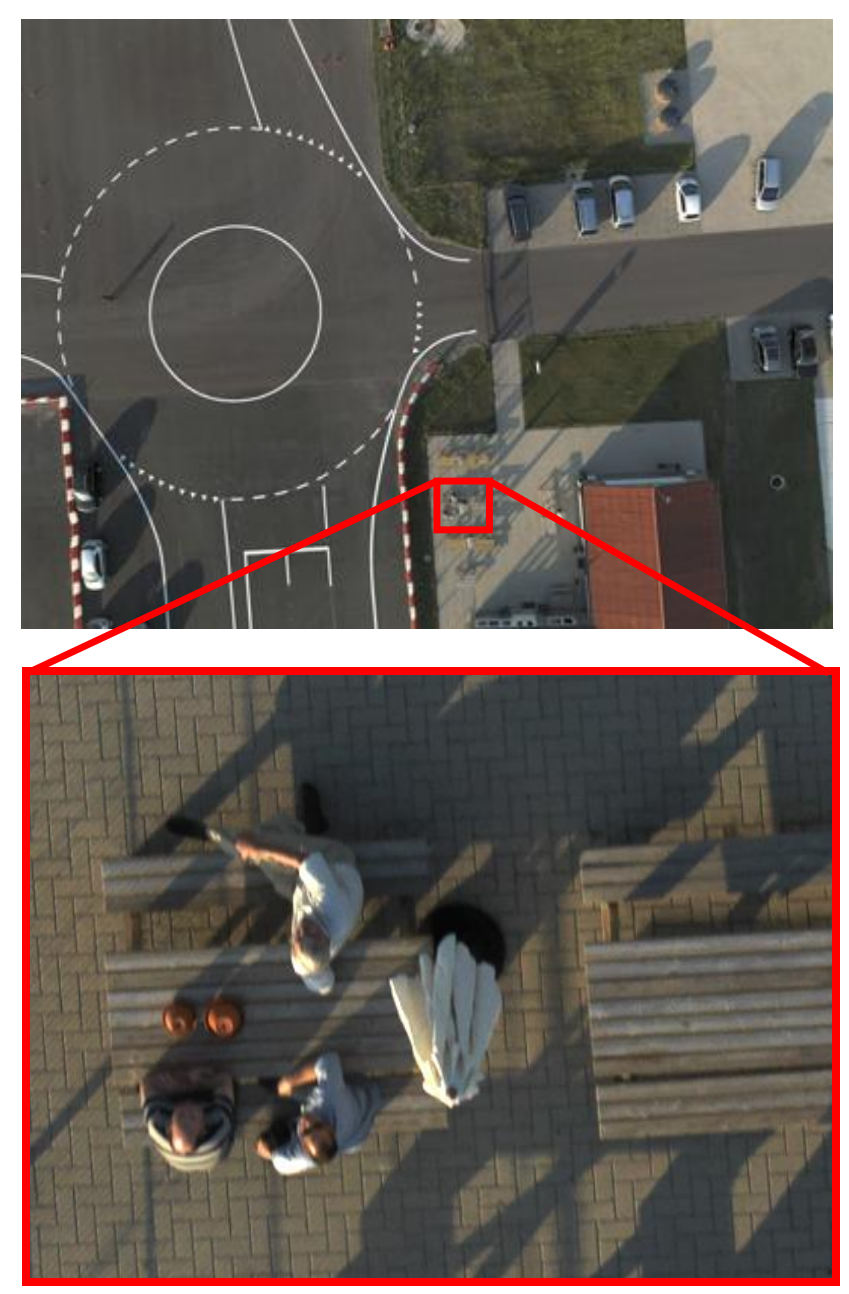

Figure 5. Entire Image (top) and Full Resolution (bottom) 
Twenty-two ground control points were accurately measured by tachymeter in $\mathrm{X}, \mathrm{Y}, \mathrm{Z}$ to validate the accuracy of the photogrammetric block. The accuracy results are expected to be presented at the workshop.

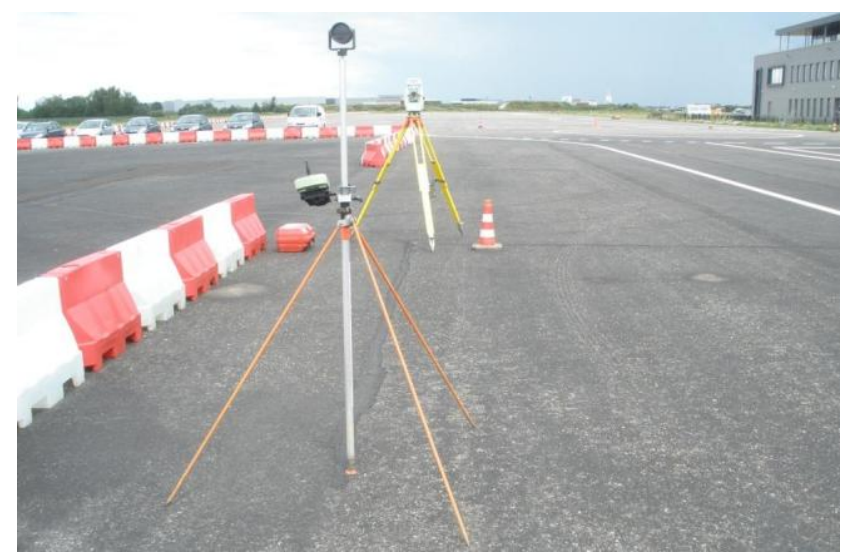

Figure 6. Ground Control Measurements

The successful integration has proven that UAS-g are capable of undertaking aerial survey flights with state-of-the-art sensors currently performed by manned aircraft. These UAS-g systems are now available for operational use.

Other state-of-the-art sensor integrations, including lidar scanner, hyperspectral camera, passive microwave radiometer, thermal infrared camera, and mini-SAR are currently being developed. It is, however, a new technology in terms of use and of end-user awareness, and this is a much bigger challenge for the UAS-g community than realizing technical capability.

\subsection{Rationale Whether to Invest in a UAS-g}

Once the end-user awareness is there, the main questions that come along with deciding whether to invest in a UAS-g are, of course, related to costs and operational use. More specifically, potential end-users wish to know more about:

$\checkmark \quad$ What are the operational costs per hour, including initial investment, maintenance, insurance;

$\checkmark$ Which cameras and sensors can be installed as far as weight and on-board power supply;

$\checkmark \quad$ What is the accuracy of the resulting geo-data;

$\checkmark$ How about crew training and number of crew;

$\checkmark \quad$ Which data link frequencies are used;

$\checkmark \quad$ Can the UAS-g be flown in strong wind and rain;

$\checkmark$ How about safety, system warranty, legislation and certification.

\section{CURRENT STATUS OF UAS LEGISLATION}

\subsection{Light UAS Legislation}

The operational and technical requirements of UAS remain the safeguard to protect people and costly sensor payload assets. An international workgroup of CAA have therefore harmonized operational regulation and certification specifications for the use of UAS in their airspace. An acceptable level of safety is to be combined with the benefits of UAS-g to collect spatial data for geo-information.

In 2007, Geocopter filed a first request for the (restricted) type certification of the GC-201. Once it was decided that the CAA of the Netherlands would accept this certification request from Geocopter, it was also decided to work together with CAA of other countries to harmonize the regulations to support the type certification and subsequent operation.

The basic regulation with which the European Aviation Safety Agency (EASA) was established dictated that for unmanned aircraft EASA is responsible for UAS over $150 \mathrm{~kg}$, and the individual European national CAA are responsible for UAS below $150 \mathrm{~kg}$. Figure 7 below shows the current participating countries in the harmonization group called JARUS.

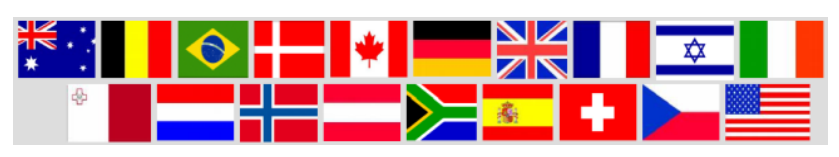

Figure 7. JARUS Countries

All rotorcraft UAS up to $150 \mathrm{~kg}$ that are commercially used or on project basis, fall under the new regulation called CS-LURS (Light Unmanned Rotorcraft System) and must therefore be approved by the national CAA (Van de Leijgraaf, 2011). The fixed wing UAS regulation is called CS-LUAS (Light Unmanned Aeroplane System).

The Light UAS regulations are divided into a Class 1, Class 2 and Class 3 approval. Table 2 below lists the main restrictive parameters of each class. In some other countries not participating in JARUS there could be other regulations and guidelines.

\begin{tabular}{|c|c|c|c|}
\hline Parameter & Class 1 & Class 2 & Class 3 \\
\hline $\begin{array}{l}\text { Visual Line } \\
\text { Of Sight } \\
\text { (VLOS) } \\
\end{array}$ & yes & yes & yes \\
\hline Radius & $500 \mathrm{~m}$ & $500 \mathrm{~m}$ & \\
\hline $\begin{array}{l}\text { Flying } \\
\text { Height AGL }\end{array}$ & $400 \mathrm{ft}$ & $400 \mathrm{ft}$ & \\
\hline Airspace & $\begin{array}{l}\text { Rural, non- } \\
\text { segregated }\end{array}$ & $\begin{array}{l}\text { Urban, non- } \\
\text { segregated or } \\
\text { segregated }\end{array}$ & $\begin{array}{l}\text { Rural, } \\
\text { segregated }\end{array}$ \\
\hline
\end{tabular}

Table 2. UAS Regulation Classes

No formal airworthiness certification is required for Class 1 and Class 3, but approval to operate is based on the technical assessment of construction and systems, and demonstration/test flights to show correct operation and crew competence. For all three classes, coordination with the local Air Traffic Service Unit (ATSU) is required. 
The formal regulation for Class 2 approval consists of:

$\checkmark$ The UAS-g must have received an Air Worthiness Certificate (AWC) issued by the national CAA;

$\checkmark$ The design of the UAS-g must have received a Type Certificate (TC) issued by the national CAA;

$\checkmark \quad$ Their pilot in command must have received a UAS pilot license (that is mainly based on the Private Pilot License);

$\checkmark \quad$ The manufacturer of the UAS must have full certificate for Design (DOA), production (POA) and maintenance (MOA).

The two main technical additions of a Class 2 approved Light UAS-g are a two-engine system and a detect-and-avoid system. The UK CAA is scheduled to publish the first draft of regulatory proposals for sense-and-avoid systems and autonomous decision-making by UAS in civil airspace. Regulation and approval Beyond Line Of Sight (BLOS) are currently under development.

The technical requirements of the AWC and TC for the Class 2 approval include thorough testing and approval of:

$\checkmark \quad$ Avionics and electrical systems;

$\checkmark$ Software;

$\checkmark$ Mechanical systems and guidance;

$\checkmark$ Strength and construction;

$\checkmark$ Engines and drive train;

$\checkmark$ Noise;

$\checkmark$ Operational flight.

As an example, the engines, drive train, and mechanical rotor head of the GC-201 have been thoroughly ground tested in highly loaded conditions as part of this certification process. Under the full load of and maximum forward speed the system has run many hours while forces and vibrations were being monitored on a balance (Figure 8).

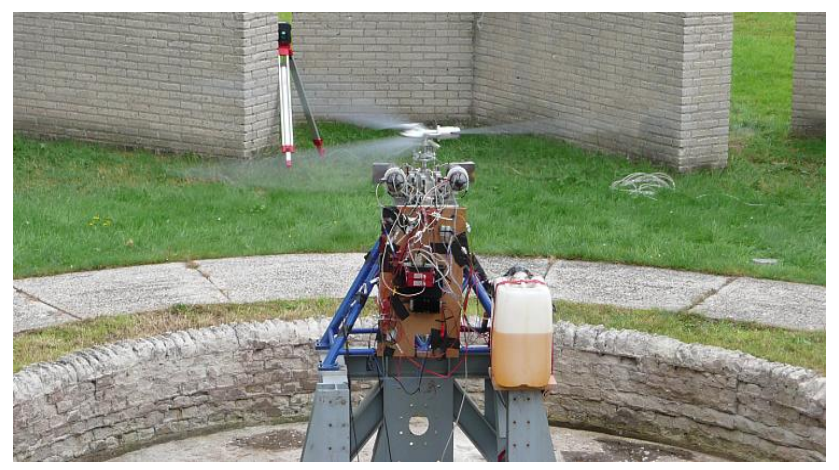

Figure 8. Endurance Test of Geocopter GC-201

\subsection{Operational Prospects of UAS-g}

Within the frame of the legislation one can now justify Light UAS-g operations for scientific applications, and for contractor supplied flight services when flying mapping projects of smaller areas of 10 up to 100 hectares, including:

$\checkmark$ Coastal zones;

$\checkmark$ Forestry;

$\checkmark$ Agriculture;

$\checkmark$ Open mines.
Sooner return on investment happens when the UAS legislation will be opening up airspace over urban areas (Class 2 approved UAS-g), to longer distances (BLOS operations), and to higher altitudes. Then scientific applications and contractor supplied flight services become feasible to the maximum extent for:

$\checkmark \quad$ Cadastral mapping of larger areas;

$\checkmark \quad$ Oil and gas pipeline monitoring;

$\checkmark$ Power line surveys;

$\checkmark$ Dike inspection;

$\checkmark$ Highway and railway mapping.

\section{CONCLUSIONS AND FUTURE WORK}

\subsection{Conclusions}

We have discussed that the current operational prospects of Light UAS-g with state-of-the-art sensors depend on four challenges: the technical capability of UAS and sensors, enduser awareness, the return on investment, and legislation.

UAS-g with state-of-the-art aerial survey sensors are now available for operational use. An example is the integration and successful automatic flight of IGI's DigiCAM with AEROcontrol on board Geocopter's GC-201. 8-mm high resolution aerial photography was captured with directgeoreferencing.

\subsection{Future Work}

Future work will focus on development of a usable detect-andavoid system (Figure 9). In addition, more state-of-the-art sensor integrations will be developed, including lidar scanner, hyperspectral camera, passive microwave radiometer, thermal infrared camera, and mini-SAR.

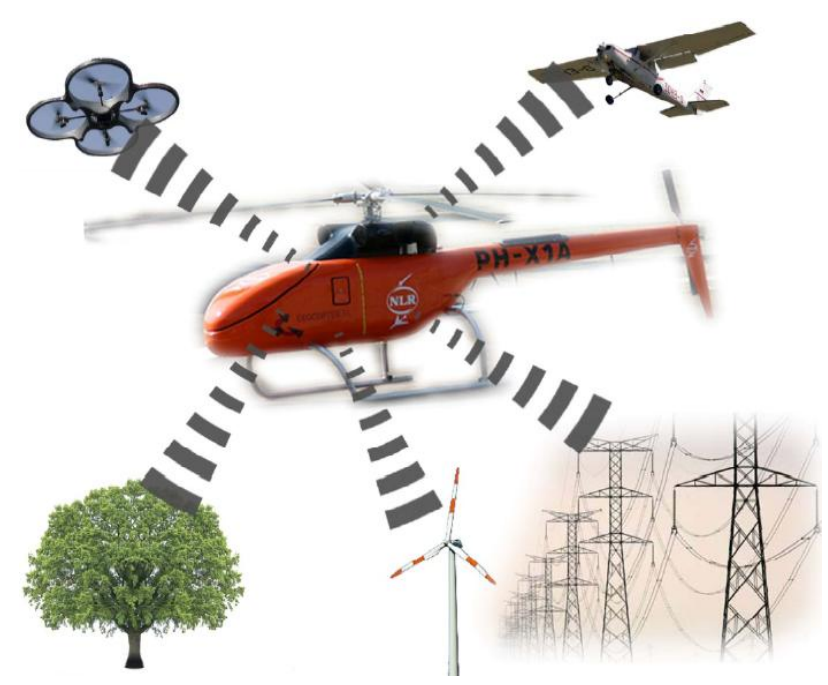

Figure 9. Detect-and-avoid System

\section{REFERENCES}

Eisenbeiss, H., 2009. UAV photogrammetry. DISS. ETH NO. 18515, doi:10.3929/ethz-a-005939264. IGP, ETH Zurich, Switzerland, Mitteilungen Nr.105, p. 235. 
International Archives of the Photogrammetry, Remote Sensing and Spatial Information Sciences, Volume XXXVIII-1/C22, 2011 ISPRS Zurich 2011 Workshop, 14-16 September 2011, Zurich, Switzerland

European Commission, 2009. Hearing on Light UAS Report Oct 2009, European Commission, Directorate-General Mobility and Transport, Directorate E, Air Transport.

Haarbrink, R., Eisenbeiss, H., 2008. Accurate DTM

Production from Unmanned Helicopter Systems.

The International Archives of the Photogrammetry, Remote Sensing and Spatial Information Sciences. Vol. XXXVII. Part B1, Beijing, China, pp. 1259-1264.

Van de Leijgraaf, R., 2011: A Coordinated International Approach to Small UAS Rulemaking. In: 2011 UAS

Yearbook, van Blyenburgh, P. (ed.), 40-41. 